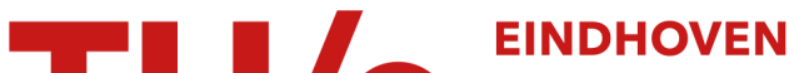 \\ UNIVERSITY OF \\ TECHNOLOGY
}

\section{Tunneling-assisted impact ionization fronts in semiconductors}

\section{Citation for published version (APA):}

Rodin, P., Ebert, U., Hundsdorfer, W., \& Grekhov, I. V. (2002). Tunneling-assisted impact ionization fronts in semiconductors. Journal of Applied Physics, 92(2), 958-964. https://doi.org/10.1063/1.1486258

DOI:

10.1063/1.1486258

Document status and date:

Published: 01/01/2002

\section{Document Version:}

Publisher's PDF, also known as Version of Record (includes final page, issue and volume numbers)

\section{Please check the document version of this publication:}

- A submitted manuscript is the version of the article upon submission and before peer-review. There can be important differences between the submitted version and the official published version of record. People interested in the research are advised to contact the author for the final version of the publication, or visit the $\mathrm{DOI}$ to the publisher's website.

- The final author version and the galley proof are versions of the publication after peer review.

- The final published version features the final layout of the paper including the volume, issue and page numbers.

Link to publication

\section{General rights}

Copyright and moral rights for the publications made accessible in the public portal are retained by the authors and/or other copyright owners and it is a condition of accessing publications that users recognise and abide by the legal requirements associated with these rights.

- Users may download and print one copy of any publication from the public portal for the purpose of private study or research.

- You may not further distribute the material or use it for any profit-making activity or commercial gain

- You may freely distribute the URL identifying the publication in the public portal.

If the publication is distributed under the terms of Article 25fa of the Dutch Copyright Act, indicated by the "Taverne" license above, please follow below link for the End User Agreement:

www.tue.nl/taverne

Take down policy

If you believe that this document breaches copyright please contact us at:

openaccess@tue.nl

providing details and we will investigate your claim. 


\title{
Tunneling-assisted impact ionization fronts in semiconductors
}

\author{
Pavel Rodin ${ }^{\text {a) }}$ \\ Institute for Theoretical Physics, Technical University Berlin, Hardenbergstrasse 36, 10623 Berlin, \\ Germany and Centrum voor Wiskunde en Informatica, P.O. Box 94079, 1090 GB Amsterdam, \\ The Netherlands \\ Ute Ebert and Willem Hundsdorfer \\ Centrum voor Wiskunde en Informatica, P.O. Box 94079, 1090 GB Amsterdam, The Netherlands \\ Igor Grekhov \\ Ioffe Physicotechnical Institute, Politechnicheskaya 26, 194021, St. Petersburg, Russia
}

(Received 27 December 2001; accepted for publication 24 April 2002)

\begin{abstract}
We discuss a type of ionization front in layered semiconductor structures. The propagation is due to the interplay of band-to-band tunneling and impact ionization. Our numerical simulations show that the front can be triggered when an extremely sharp voltage ramp $(\sim 10 \mathrm{kV} / \mathrm{ns})$ is applied in reverse direction to a $\mathrm{Si} p^{+}-n-n^{+}$structure that is connected in series with an external load. The triggering occurs after a delay of 0.7 to $0.8 \mathrm{~ns}$. The maximal electrical field at the front edge exceeds $10^{6} \mathrm{~V} / \mathrm{cm}$. The front velocity $v_{f}$ is 40 times faster than the saturated drift velocity $v_{s}$. The front passes through the $n$-base with a thickness of $100 \mu \mathrm{m}$ within approximately $30 \mathrm{ps}$, filling it with dense electron-hole plasma. This passage is accompanied by a voltage drop from $8 \mathrm{kV}$ to a voltage in the order of $10 \mathrm{~V}$. In this way a voltage pulse with a ramp up to $500 \mathrm{kV} / \mathrm{ns}$ can be applied to the load. The possibility to create a kilovolt pulse with such a voltage rise rate sets new frontiers in pulse power electronics. (C) 2002 American Institute of Physics. [DOI: 10.1063/1.1486258]
\end{abstract}

\section{INTRODUCTION}

Impact ionization and tunneling (Zener breakdown) are the two most fundamental mechanisms capable of creating high concentrations of free carriers in a semiconductor within a picosecond time interval. The respective threshold electrical fields differ by almost one order of magnitude, e.g., $2 \times 10^{5} \mathrm{~V} / \mathrm{cm}$ and $10^{6} \mathrm{~V} / \mathrm{cm}$ for impact and tunneling ionization in $\mathrm{Si}$, respectively. ${ }^{1,2}$ Avalanche impact ionization can be easily achieved by applying a sufficiently high external electrical field. This process underlies the operation of many semiconductor devices such as avalanche transistors, IMPATT and TRAPATT diodes, etc. ${ }^{1-3}$ The most interesting scenario corresponds to the propagation of a superfast ionization front: a narrow impact ionization region travels from the cathode to the anode with a velocity $v_{f}$ much higher than the saturated drift velocity $v_{s}$, leaving a high density plasma behind. ${ }^{4-9}$ In contrast to impact ionization, direct tunneling of electrons from the valence to the conductance band is hard to achieve in the bulk of uniformly doped semiconductor layers: while the applied external voltage is being increased, impact ionization typically sets in first, inducing an avalanche multiplication of free carriers. This causes the conductivity to increase and prevents further increase of the applied voltage. For this reason, tunneling ionization is generally assumed to be relevant only in heavily doped $p-n$ junctions due to the strong internal electrical fields. ${ }^{1}$

In this article we demonstrate that the threshold of tunneling ionization in the bulk of a Si $p^{+}-n-n^{+}$structure can

a) On leave from Ioffe Physicotechnical Institute, Politechnicheskaya 24, 194021, St. Petersburg, Russia; electronic mail: rodin@physik.tu-berlin.de be reached under the same experimental conditions as for triggering impact ionization fronts. ${ }^{6-9}$ Typically an ionization front is triggered by applying a voltage pulse with a steep slope $(\geqslant 1 \mathrm{kV} / \mathrm{ns})$ to the $p^{+}-n-n^{+}$structure in reverse direction. ${ }^{6,9,10}$ In structures with kilovolt $p-n$ junctions and large cross-sections, this process is used for sharpening electrical pulses. ${ }^{9,11-13}$ This technique allows one to reach voltage ramps with slopes of up to $10 \mathrm{kV} / \mathrm{hs}$, the state of the art in modern pulse power electronics. We demonstrate, that when such a sharp ramp $A \sim 10 \mathrm{kV} / \mathrm{ns}$ is applied to a fully depleted reversely biased $\mathrm{Si} p^{+}-n-n^{+}$structure, the threshold of tunneling ionization $\sim 10^{6} \mathrm{~V} / \mathrm{cm}$ is reached after less than $1 \mathrm{~ns}$, which turns out to be faster than the initiation of avalanche impact ionization. The resulting breakdown takes the form of an ionization front that propagates due to the combined effect of tunneling and impact ionization. Compared to the traditional impact ionization fronts in pulse sharpening diodes ${ }^{6-9,11,12}$ and TRAPATT diodes, ${ }^{4,5}$ these tunneling-assisted impact ionization fronts are expected to be much faster and generate higher plasma concentrations. Their practical application may set new frontiers in pulse power electronics.

\section{THE MODEL}

We consider a Si $p^{+}-n-n^{+}$structure with sharp $p^{+}-n$ and $n-n^{+}$transitions and the following parameters: the width of the $n$-base is $W=100 \mu \mathrm{m}$, the cross-section area is $S=0.002 \mathrm{~cm}^{2}$, the dopant concentrations are $N_{d}$ $\approx 10^{14} \mathrm{~cm}^{-3}$ in the $n$-base and $N_{a, d} \approx 10^{19}-10^{20} \mathrm{~cm}^{-3}$ in the contact $p^{+}$- and $n^{+}$-layers, respectively. These parameters correspond to a typical Si power diode with a stationary 


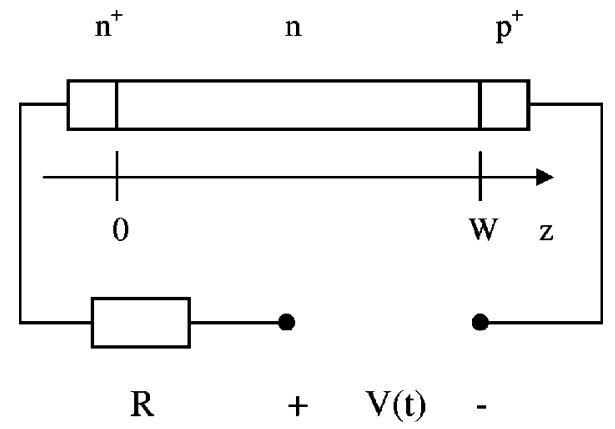

FIG. 1. Sketch of the $p^{+}-n-n^{+}$structure operated in an external circuit with load resistance $R$.

breakdown voltage $\sim 1.5 \mathrm{kV} .{ }^{1}$ We choose the initial bias of $V_{0}=1 \mathrm{kV}$, closely below the voltage of stationary avalanche breakdown. For this bias, the $n$-base is fully depleted from majority carriers (electrons) and equilibrium minority carriers (holes).

The device is connected to a voltage source $V(t)$ in series with a load resistance $R=50 \Omega$ as sketched in Fig. 1 . Since it is convenient to work with a positive electrical field $E$ for the reverse bias, we put the $n-n^{+}$junction on the left-hand side at $z=0$ and the $p^{+}-n$ junction on the righthand side at $z=W$. The voltage $V(t)$ applied to the structure and the load typically represents a sinusoidal pulse $V(t)$ $=V_{0}+V_{1} \sin (2 \pi t / T)$. We assume that the period $T$ of this pulse is much larger than the duration of the ionization breakdown and approximate the applied voltage by the linear function

$$
V(t)=V_{0}+A t, \quad A=\frac{2 \pi V_{1}}{T},
$$

where $A$ is the voltage ramp parameter. Hereinafter we choose $A=10 \mathrm{kV} / \mathrm{ns}$. The voltage on the device is denoted as $U(t)$ and related to $V(t)$ through the Kirchhoff equation $V$ $=U+R I$, where $I$ is the total current.

We use a minimal model which accounts only for the basic transport processes, for band-to-band impact ionization and tunneling ionization. The continuity equations for electrons and holes $n$ and $p$ are written in one-dimensional approximation

$$
\begin{aligned}
& \partial_{t} n-\partial_{z}\left[v_{n}(|E|) n\right]=G(n, p,|E|), \\
& \partial_{t} p+\partial_{z}\left[v_{p}(|E|) p\right]=G(n, p,|E|),
\end{aligned}
$$

and complemented by the Poisson equation

$$
\partial_{z} E=\frac{q}{\epsilon \epsilon_{0}}\left[p-n+N_{d}^{+}(z)-N_{a}(z)^{-}\right],
$$

where all dopants are ionized, and the Kirchhoff equation. We assume drift-dominated transport and approximate the carrier velocities as ${ }^{14}$

$$
v_{n}(|E|)=v_{s} \frac{|E|}{E_{s n}+|E|}, \quad v_{p}(|E|)=v_{s} \frac{|E|}{E_{s p}+|E|},
$$

where

$$
v_{s}=10^{7} \mathrm{~cm} / \mathrm{s},
$$

$$
E_{s n}=8.0 \times 10^{3} \mathrm{~V} / \mathrm{cm}, \quad E_{s p}=2.32 \times 10^{4} \mathrm{~V} / \mathrm{cm} .
$$

Since the ionization processes are fast and develop in the bulk of the device, we solve these equations in the $n$-base only. The different effect of $p^{+}-n$ and $n-n^{+}$junctions on electron and hole concentrations in the $n$-base is modeled by mixed boundary conditions: $\partial_{z} n=0, p=0$ at $z=0$ and $\partial_{z} p$ $=0, n=0$ at $z=W \cdot{ }^{15}$ The generation term $G(n, p,|E|)$ contains band-to-band tunneling and impact ionization terms:

$$
G(n, p,|E|)=G_{T}(|E|)+G_{I}(n, p,|E|) .
$$

The tunneling term $G_{T}(|E|)$ models electron tunneling from the valence band to the conduction band ${ }^{16}$

$$
\begin{aligned}
& G_{T}(|E|)=\alpha_{T} E^{2} e^{-b_{T} /|E|}, \\
& \alpha_{T}=\frac{q^{2}}{3 \pi^{2} \hbar^{2}} \sqrt{\frac{2 m}{E_{g}}}, \quad b_{T}=\frac{\pi}{4 q \hbar} \sqrt{2 m E_{g}^{3}},
\end{aligned}
$$

where $q$ and $m$ are electron charge and effective mass, respectively, $E_{g}$ is the band gap, and $\hbar$ is Planck's constant. The impact ionization term $G_{I}(n, p,|E|)$ is chosen as

$$
\begin{aligned}
& G_{I}(n, p,|E|)= \alpha_{n}(|E|) v_{n}(|E|) n \Theta\left(n-n_{\text {cut }}\right) \\
&+\alpha_{p}(|E|) v_{p}(|E|) p \Theta\left(p-p_{\text {cut }}\right), \\
& \alpha_{n}(|E|) \equiv \alpha_{n s} e^{-b_{n} /|E|}, \quad \alpha_{p}(|E|) \equiv \alpha_{p s} e^{-b_{p} /|E|},
\end{aligned}
$$

where the impact ionization coefficients and the characteristic fields are given by ${ }^{17}$

$$
\begin{aligned}
& \alpha_{n s}=7.4 \times 10^{5} \mathrm{~cm}^{-1}, \quad \alpha_{p s}=7.25 \times 10^{5} \mathrm{~cm}^{-1}, \\
& b_{n}=1.1 \times 10^{6} \mathrm{~V} / \mathrm{cm}, \quad b_{p}=2.2 \times 10^{6} \mathrm{~V} / \mathrm{cm},
\end{aligned}
$$

and $\Theta(x)$ is the step function. The cutoffs $n_{\text {cut }}$ and $p_{\text {cut }}$ have been introduced in Ref. 9 to mimic the discreteness of the charge carriers. The purpose is to exclude unphysical ionization avalanches initiated by tiny fractions of electrons or holes that can cause premature triggering of the front in the simulations. We chose $n_{\text {cut }}=p_{\text {cut }}=10^{9} \mathrm{~cm}^{-3}$ in the simulations and discuss the effect of this and other choices in Sec. $\mathrm{V}$ and Fig. 6.

Since we shall investigate processes on a subnanosecond time scale, we neglect all types of recombination and thermal generation. We also assume that the $n$-base is free of deeplevel defects or parasitic impurities that can assist tunneling or serve as deep-level electron traps capable to release electrons or holes in high electric field. ${ }^{18,19}$ We discuss these model assumptions in detail in Secs. IV and V.

We use a uniform space-time grid with the number of points of the order of several thousands both in time and space. The spatial discretization is based on a conservative formulation, in terms of fluxes describing the inflow and outflow over cells $(x-\Delta x / 2, x+\Delta x / 2)$, where $\Delta x$ is the grid width in space. Whereas the diffusive fluxes have been approximated in a standard fashion ${ }^{20}$ with second order accuracy, for the advective fluxes a third order upwind biased formula has been chosen in order to reduce the numerical oscillations. Time discretization is based on a second order backward differentiation formula. The temporal backward differentiation formula gives an implicit system that is solved at each time step. For reasons of accuracy the time step $\Delta t$ is 

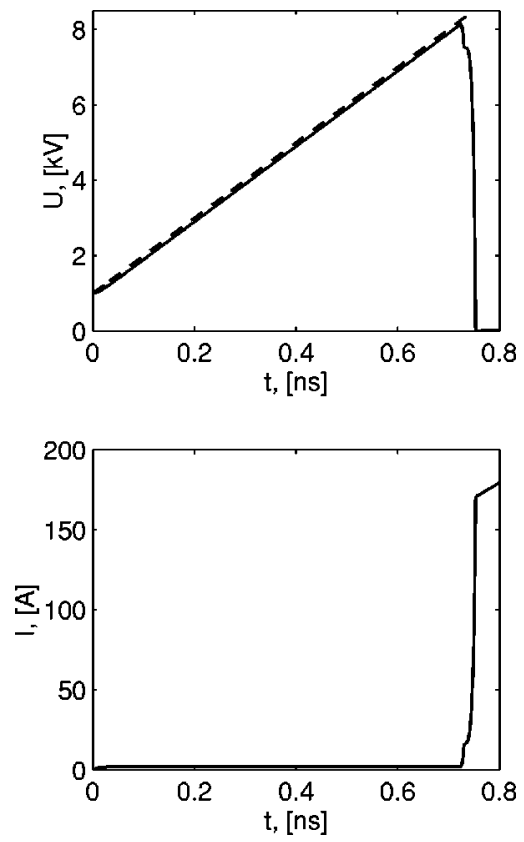

FIG. 2. Voltage drop across the structure $U(t)$ (solid line in the upper panel) and total current $I(t)=S J(t)$ (in the lower panel) during the switching process. The dashed line in the upper panel denotes the externally applied voltage $V(t)$. The quantities shown are related through Ohm's law $V=U$ $+R I$. Parameters: $W=100 \mu \mathrm{m}, S=0.002 \mathrm{~cm}^{2}, N_{d}=10^{14} \mathrm{~cm}^{-3}, N_{a}=0$, $V_{0}=1 \mathrm{kV}, A=10 \mathrm{kV} / \mathrm{ns}, R=50 \Omega, n_{\text {cut }}=p_{\text {cut }}=10^{9} \mathrm{~cm}^{-3}$.

chosen small compared to $\Delta x / v_{s}$, where $v_{s}$ is the upper bound of the drift velocity, and with such small step size that the implicit system can be solved by a straightforward functional iteration. Details on these spatial and temporal discretizations can be found in Ref. 21.

\section{NUMERICAL SOLUTIONS}

The basic features of the numerical solutions are summarized in Figs. 2 and 3. They show the external characteristics of the transient and the internal dynamics, respectively. The voltage on the device first increases and reaches the maximal value $8 \mathrm{kV}$, several times higher than the voltage of stationary breakdown. During this stage, the electrical field in the structure increases as follows:

$$
E(x, t) \approx \frac{V(t)}{W}-\frac{b W}{2}+b x, \quad b \equiv \frac{q N_{d}}{\epsilon \epsilon_{0}},
$$

in the same manner as in the TRAPPAT diode. ${ }^{1,5}$ [Here we assume that the displacement current is small and hence $V(t) \approx U(t)$.] Though the electrical field $E$ at the right boundary exceeds the effective threshold of impact ionization $2 \times 10^{5} \mathrm{~V} / \mathrm{cm}$ already at $t>30 \mathrm{ps}$, impact ionization does not develop due to the absence of initial carriers. At $t$ $\approx 720 \mathrm{ps}$ the electrical field at the right boundary $x=W$, near the $p^{+}-n$ junction, becomes sufficient for tunneling of electrons from the valence to the conduction band [Fig. 3(a), curve 1]. At this time, the field is above the threshold of impact ionization in the whole $n$-base, so impact ionization starts as soon as electrons and holes are supplied by tunneling. The rate of impact ionization increases with concentration and eventually overheads tunneling at the carrier density (a)
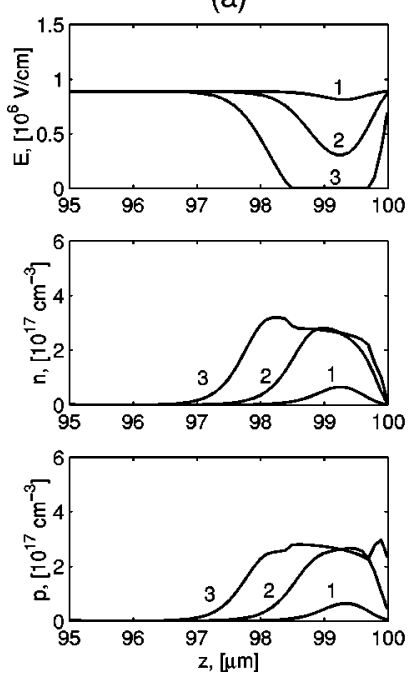

(b)
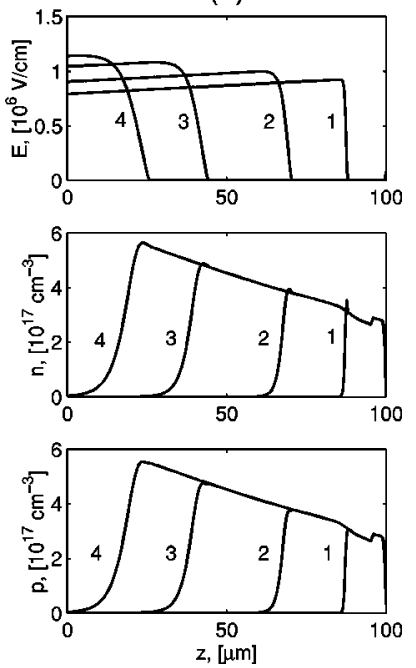

FIG. 3. The internal dynamics leading to the external characteristics of Fig. 2. Shown are the spatial profiles of the electrical field $E(x, t)$ and electron and hole concentrations $n(x, t), p(x, t)$ in the $n$-base $(0 \leqslant z \leqslant W=100 \mu \mathrm{m})$ at different times: (a) nucleation of electron-hole plasma and triggering of the impact ionization front at times $t=725,726,727$ ps (curves 1,2,3); (b) propagation of the tunneling-assisted ionization front at $t$ $=735,745,750,752$ ps (curves 1,2,3,4).

$n \sim p \sim 10^{13} \mathrm{~cm}^{-3}$ (see Fig. 4). The rapid ionization process near the right boundary forms an initial nucleus of dense electron-plasma with concentration $n, p \sim 2 \times 10^{17} \mathrm{~cm}^{-3}$ that is capable to fully screen the electrical field [Fig. 3(a), curves $2,3]$. The screening is accomplished at $t=750 \mathrm{ps}$. It is accompanied by a fast drop of the voltage on the device and an increase of current in the circuit (Fig. 2). This drop corresponds to the first step in the falling $U(t)$ curve.

Consecutively, the highly conducting plasma region expands to the left in the form of a superfast ionization front [Fig. 3(b)]. The velocity of the front $v_{f}$ is approximately 40 times faster than the saturated drift velocity $v_{s}$ of the individual carriers; hence the carrier motion is negligible. The front propagates due to the combined effect of tunneling and impact ionization, followed by Maxwell relaxation in the generated plasma and consecutive electric screening. Snapshots of the field and concentration profiles together with respective generation rates are shown in Fig. 5. Here tunneling generates initial carriers in the high field region at the

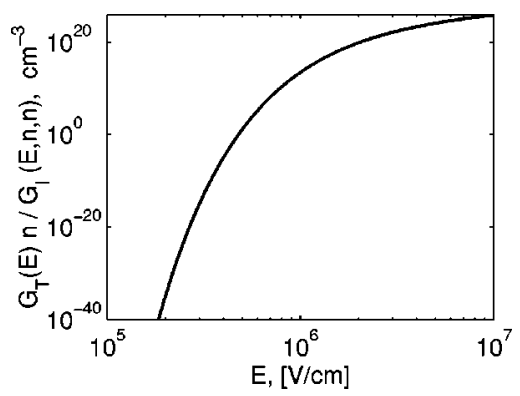

FIG. 4. Shown is the carrier concentration $n(E)$ for which impact ionization and tunneling ionization are of equal strength. This concentration can be expressed as the ratio $G_{T}(E) n / G_{I}(E, n, n)$, where we assumed $n=p$ and neglected the cutoffs in Eq. (9). 

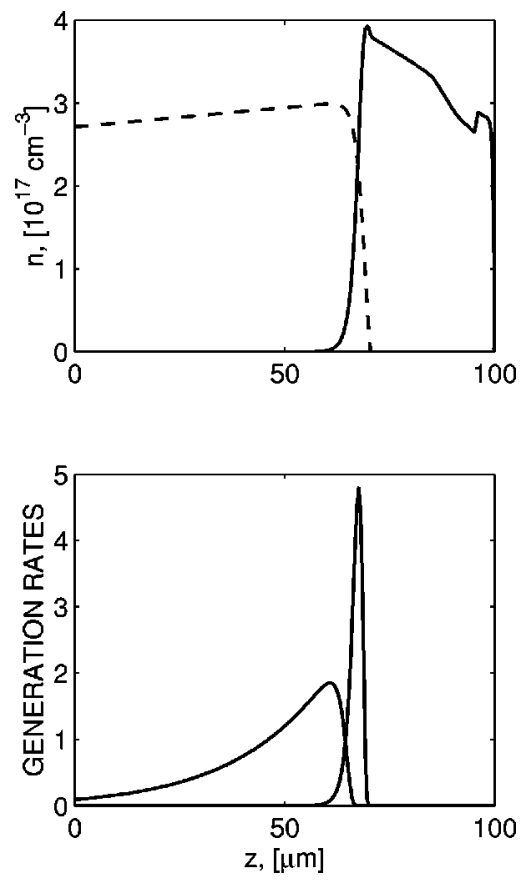

FIG. 5. The inner structure of the tunneling-assisted front (at $t=740 \mathrm{ps}$ ). The upper panel shows the electron concentration (solid line) and the electrical field profile (dashed line, in arbitrary units). The lower panel shows spatial profiles of the tunneling generation rate $G_{T}$ (curve 1, one unit corresponds to $10^{27} / \mathrm{s} \mathrm{cm}^{3}$ and the impact ionization rate $G_{I}$ (curve 2, one unit corresponds to $10^{36} / \mathrm{s} \mathrm{cm}^{3}$ ). Note the different scales of $G_{T}$ and $G_{I}$.

edge of the ionization front. These carriers are multiplied further by impact ionization. A narrow region of strong impact ionization is localized at the edge of the visible concentration front. The rate of impact ionization in this region is by many orders of magnitude higher than the rate of tunneling ionization. Therefore impact ionization dominates the overall increase of the concentration. However, the superfast propagation would not be possible without the initial carriers generated by tunneling. Hence both tunneling and impact ionization are essential for the propagation mechanism. This type of an ionization front can be called a tunneling-assisted impact ionization front.

As the front propagates, the current $I$ increases and the voltage on the device $U$ decreases due to the interaction with the external load. The maximum value of the electrical field and the front velocity slightly increase during the passage of the front [Fig. 3(b)]. As the front comes closer to the left boundary, the field in the high-field region of the $n$-base increases and the region of tunneling impact ionization becomes wider. This effect also contributes to the acceleration of the front, and causes the front interface to become smoother (compare curves 1,2 and 3,4 in Fig. 3). Eventually, the tunneling process becomes efficient in the whole region between the moving front and the $n^{+}$-contact. As a result, the front ceases to propagate. Rather, the rest of the $n$-base breaks down by nearly uniform impact ionization.

The switching takes $30 \mathrm{ps}$ and fills the $n$-base with electron-hole plasma of concentration $\sim 5 \times 10^{17} \mathrm{~cm}^{-3}$. The voltage drops from $8 \mathrm{kV}$ to the residual value of $10 \mathrm{~V}$, applying a voltage ramp with an average slope $\sim 300 \mathrm{kV} / \mathrm{ns}$ to the load. The falling part of the $U(t)$-dependence in Fig. 2 has a plateau that reflects the transition from the formation of the initial nucleus of electron-hole plasma to the stage of front propagation. During the front propagation stage, the voltage drops from $\sim 7.4 \mathrm{kV}$ to $10 \mathrm{~V}$ within less than $15 \mathrm{ps}$. Hence the "effective" voltage slope is much higher than the average one and reaches $500 \mathrm{kV} / \mathrm{ns}$.

\section{THE EFFECT OF RANDOM THERMAL GENERATION}

The triggering of the tunneling-assisted impact ionization front might fail due to the thermal generation of free carriers in the depleted layer. If during an early stage of development, thermal carriers lead to an avalanche breakdown and to the formation of local conducting channels between the $p^{+}$- and $n^{+}$-layers, the conductance might increase and prevent the voltage $U(t)$ from increasing further. In this case, the front cannot start. Therefore the related time scales and their temperature dependence have to be estimated.

The characteristic rate of thermal generation of electron-hole pairs can be deduced from the value of the leakage current density. It is $\sim 10^{-7} \mathrm{~A} / \mathrm{cm}^{2}$ at room temperature. ${ }^{6,9}$ Hence on average, one thermal electron-hole pair per ns is generated in the whole volume of the $n$-base. Depending on the position of the generation, the carriers need up to 1 ns to reach the edge of the $n$-base of length 100 $\mu \mathrm{m}$, when they move with saturated drift velocity $10^{7} \mathrm{~cm} / \mathrm{s}$. If the field is still low, they will simply leave the base, but if they are generated in a region with $E>2 \times 10^{5} \mathrm{~V} / \mathrm{cm}$ or pass through such a region, they will create an impact ionization avalanche and possibly a successive streamer on their path. After $0.7 \mathrm{~ns}$, the tunneling-assisted impact ionization front leads to a rapid voltage drop. Though the probability that one ionization avalanche will be initiated during the delay time is close to one, this avalanche in most cases cannot form a conducting channel before the front is triggered. The probability for more avalanches decreases exponentially with their number.

However, even if a single conducting channel has developed, the ratio between its cross section and the cross section of the system is too small to influence the triggering of the superfast front. Indeed, an avalanche triggered by a single electron or hole extends in the direction of the applied field with drift velocity $v_{s}$ and spreads in the lateral direction due to diffusion (see, e.g., Ref. 22). Since the latter process is much slower than the first one, the width of the avalanche is typically much smaller than its length, which is of order of $100 \mu \mathrm{m}$ in our case. A reasonable upper bound for the maximum width is of order of $10 \mu \mathrm{m} .{ }^{23}$ This estimate is consistent with diameters of local switching channels observed experimentally under similar though slightly different experimental conditions. ${ }^{24-26}$ Thus the cross-section of the local switching channel is less than $1 / 1000$ of the total crosssection of the semiconductor structure. Assuming that concentrations in a channel are limited by Auger recombination at the level $n, p \sim 10^{18} \mathrm{~cm}^{-3}$ and high-field transport with saturated velocities $v_{s}$, we evaluate the maximum current that can flow through such channel as $\sim 1 \mathrm{~A}^{27}$ This is comparable to the displacement current in the structure during 
the delay stage, and far too small to prevent the further increase of the voltage on a structure with large cross-section.

The generation rate depends exponentially on the temperature: ${ }^{1}$

$$
\nu(T)=\nu\left(T_{0}\right) \exp \left(\frac{E_{g}}{k T_{0}}-\frac{E_{g}}{k T}\right),
$$

where $k$ is Boltzmann's constant and $T_{0}$ is room temperature. For $T=70 \mathrm{~K}$ and $T=400 \mathrm{~K}$, we find characteristic times of $\tau \sim 10 \mathrm{~ms}$ and $\tau \sim 1 \mathrm{ps}$, respectively. Hence the triggering mechanism is very sensitive to the temperature of the structure: cooling the sample is favorable whereas triggering of tunneling-assisted fronts is likely to be impossible at high temperatures. $^{28}$

We conclude that the tunneling-assisted impact ionization front can be triggered only if the applied voltage increases sufficiently fast and the threshold of tunneling ionization is reached faster than $1 \mathrm{~ns}$. At room temperature and below, the triggering mechanism is robust with respect to random initiation of impact ionization avalanches by thermal carriers. This conclusion is also supported by experimental data for common impact ionization fronts: ${ }^{6,9}$ for comparable experimental setup and the lower voltage ramp of $A$ $\sim 1 \mathrm{kV} / \mathrm{ns}$, the delay in deterministic triggering of the impact ionization front could be as large as $3 \mathrm{~ns}$.

\section{LIMITATIONS OF THE DRIFT-DIFFUSION MODEL}

In this section, we discuss the applicability of the minimal drift-diffusion model to the rapid high-field process described in Sec. III. Let us first briefly summarize the characteristic scales of the obtained numerical solution: the front length is $l_{f} \sim 3 \mu \mathrm{m}$, the front velocity $v_{f} \sim 4 \times 10^{8} \mathrm{~cm} / \mathrm{s}$, the total switching time $\sim 30 \mathrm{ps}$, the concentration behind the front $\sim 5 \times 10^{17} \mathrm{~cm}^{-3}$, and the current density $J$ $\sim 10^{5} \mathrm{~A} / \mathrm{cm}^{2}$.

Relaxation time and electromagnetic propagation time. A simple evaluation in the spirit of Drude theory and based on the low-field mobility, estimates the upper bound for the electron mean free path and the lower bound for the electron momentum relaxation time as $20 \mathrm{~nm}$ and $0.2 \mathrm{ps}$, respectively. The electromagnetic propagation time is $W / c=0.5 \mathrm{ps}$, where $c$ is the speed of light. These scales are considerably smaller than the respective scales of the process under study.

Recombination. In $\mathrm{Si}$ for concentrations $n=p$ $=10^{18} \mathrm{~cm}^{-3}$, the inverse rate of Auger recombination is $\sim 10 \mu \mathrm{s}$; the thermal recombination lifetime is also of the order of microseconds. ${ }^{29}$ Thus recombination can be neglected.

Electron-hole scattering. For current densities above $10^{2} \mathrm{~A} / \mathrm{cm}^{2}$, electron-hole scattering, which is not accounted for in our model, becomes important. ${ }^{30}$ Due to the electronhole scattering, the resistivity $\rho$ of the dense plasma behind the front substantially increases. For $J \sim 10^{3} \mathrm{~A} / \mathrm{cm}^{2}$ and $n, p \sim 10^{18} \mathrm{~cm}^{-3}$ we estimate the order of the magnitude as $\rho \sim 1 \Omega \mathrm{cm} .{ }^{30}$ Hence the voltage drop across the $n$-base after switching can be estimated as $J \varrho W \sim 10 \mathrm{~V}$. This value should be added to the $10 \mathrm{~V}$ obtained in our simulations. (Note that in our model the residual voltage is mostly due to

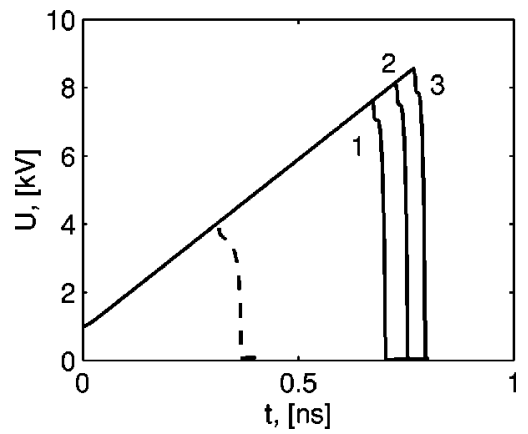

FIG. 6. Voltage drop across the structure $U(t)$ calculated for different cutoffs $n_{\text {cut }}=p_{\text {cut }}=10^{8}, 10^{9}, 5 \times 10^{9} \mathrm{~cm}^{-3}$ (curves $1,2,3$, respectively). The dotted line shows the unphysical premature switching in a low electrical field obtained for $n_{\text {cut }}=p_{\text {cut }}=0$.

the recovery of the electrical field on the $p^{+}-n$ junction during the front propagation.) This difference of $10 \mathrm{~V}$ is negligible compared to the kilovolt voltage drop during the front passage, and does not change other characteristics of the switching process.

Continuum approximation. The continuum treatment of electron and hole concentrations is the major limitation of the standard drift-diffusion model in application to the present problem. Both tunneling and impact ionization terms ignore the discrete nature of the ionization process: the tunneling term predicts a small but steady increase of the concentration even for electrical fields that are far below the effective threshold of tunneling ionization. In turn, the impact ionization generation term (10) models the multiplication of any concentration of free carriers, even if these concentrations physically correspond to a tiny fraction of an electron or hole in the whole volume of the device. Hence the unreflected use of a continuum approximation leads to physically meaningless results: triggering of the front is observed at very low electrical fields due to the multiplication of unphysically small concentrations of free carriers. Such an unphysical solution that predicts premature triggering of the front is depicted by the dashed line in Fig. 6: the front is triggered at $t \approx 300 \mathrm{ps}$ when the maximum electrical field is not more than $E(W, t)=5 \times 10^{5} \mathrm{~V} / \mathrm{cm}$, which is too low for tunneling ionization.

These unphysical solutions can be eliminated by introducing the cutoffs for low concentrations in the impact ionization generation term (9). In Fig. 6, we show the transient characteristics for different cutoffs $n_{\text {cut }}$. In a wide range of physically meaningful parameter values of $n_{\text {cut }}$, the front triggering and propagation is qualitatively the same. However, the time delay in the front triggering somewhat depends on the cutoff level, thus making the continuum model unsuitable for accurate quantitative predictions. The delay time shifts by approximately $50 \mathrm{ps}$ when the cutoff level changes by one order of magnitude in both directions, whereas the triggering time remains approximately the same. A quantitative analysis would demand a more elaborate stochastic microscopic model. 


\section{DISCUSSION}

Tunneling-assisted impact ionization fronts are quite similar to the well studied case of superfast impact ionization fronts that underly the operation of TRAPPAT diodes ${ }^{4,5}$ and sharpening diodes. ${ }^{6,7,9,11,12}$ In both cases we deal with a collective phenomenon of superfast propagation. It is based on avalanche multiplication of the already existing carriers due to impact ionization in a finite narrow region of the device, followed by screening of the electrical field due to Maxwell relaxation in the adjacent spatial region. The important difference is the source of the free carriers that initiate the avalanche impact ionization process. TRAPATT-like fronts propagate into a depleted $n$-base with a certain concentration of initial carriers, often referred to as "pre-ionization." 31,32 The physical mechanism that creates pre-ionization then is unrelated to the front propagation mechanism, e.g., in the case of the TRAPPAT diode, these are nonequilibrium carriers left behind after the previous front passage. In the case under consideration here, there is no pre-ionization and the initial carriers are generated during the front passage by tunneling in a region just ahead of the impact ionization front. Hence tunneling and impact ionization coherently cooperate in the superfast propagation of the tunneling-assisted impact ionization front.

For impact ionization fronts that propagate into homogeneously pre-ionized media, an essential ingredient is the spatial profile of the electrical field: the field should be below the threshold of impact ionization at a certain distance from the front. ${ }^{5,31,32}$ This keeps the "active" region, where the impact ionization develops, finite and prevents a quasiuniform blowup of the concentration in the whole sample. For ionization fronts in $p^{+}-n-n^{+}$structures, this profile is due to the doping of the $n$-base that gives a slope of the electrical field $q N_{d} / \epsilon \epsilon_{0}$ in the depleted layer. In contrast, for the tunneling-assisted front, the electrical field is well above the threshold of impact ionization in the whole $n$-base. The size of the active region is controlled by the threshold of tunneling ionization. This allows for much higher electrical fields, increasing the front velocity and the concentration of the generated plasma by orders of magnitude.

The possibility of tunneling ionization fronts has been discussed before in Refs. 33 and 34. The theoretical investigation in Ref. 34 takes only tunneling ionization into account, assuming that in high fields the impact ionization component can be neglected. Our results show that this is not possible for electrical fields of the order of $10^{6} \mathrm{~V} / \mathrm{cm}$ : the importance of impact ionization increases with concentration and dominates tunneling ionization already for concentrations of free carriers as low as $n, p>10^{13} \mathrm{~cm}^{-3}$, as can be read from Fig. 4. Thus impact ionization is the dominant mechanism of free carrier generation even in the range of fields where tunneling is possible, after a sufficient carrier concentration is reached. Moreover, simulations with the impact ionization term set to zero show that the tunneling ionization alone does not lead to front propagation; rather the breakdown of the sample becomes quasi-uniform. We suggest the following explanation for this observation: the appearance of the traveling front solutions is known to be due to an autocatalytic dependence of the impact ionization generation rate on the concentration of initial carriers. ${ }^{5,31,32,35}$ It leads to the exponentially fast increase of the concentration with time in a given electrical field. The rate of tunneling ionization lacks this auto-catalytic dependence on the concentration, predicting an algebraically slow growth of concentration with time. We emphasize that a propagating ionization front generates much higher carrier concentrations than quasi-uniform breakdown. This is because during the front propagation the impact ionization efficiently develops in a high field region that passes through the device, leaving a highly ionized plasma behind. In contrast, for uniform breakdown, an increase of concentration is immediately followed by a decrease of electrical field due to the coupling to the external load.

\section{SUMMARY}

We have described the theory of a type of ionization front in layered Si semiconductor structures. These are superfast tunneling-assisted impact ionization fronts that propagate due to the coherent effect of tunneling and impact ionization. The front propagates into the fully depleted $n$-base of a $p^{+}-n-n^{+}$structure. The region of tunneling ionization in electrical fields higher than $10^{6} \mathrm{~V} / \mathrm{cm}$ has a characteristic length of $10 \mu \mathrm{m}$. The generated carriers initiate an avalanche impact ionization process that becomes much more efficient than tunneling as concentration increases. The concentration front has a characteristic width of several micrometers. Within this length the concentration of free carriers increases to the level of $n, p>5 \times 10^{17} \mathrm{~cm}^{-3}$. Maxwell relaxation in the generated electron-hole plasma leads to the full screening of the applied electrical field. The front propagation is a collective phenomenon based on ionization and screening, and its velocity $v_{f}$ is not limited by the high-field drift velocity of individual carriers $v_{s}$. We observe $v_{f}$ $\approx 40 v_{s}$ in our simulations.

The front triggering becomes possible if the threshold of tunneling ionization $10^{6} \mathrm{~V} / \mathrm{cm}$ is reached after a delay shorter than $1 \mathrm{~ns}$. This ensures that random thermal ionization in the depleted layer does not spoil the triggering. This condition is met at room and lower temperatures when a voltage pulse with a slope $\sim 10 \mathrm{kV} / \mathrm{ns}$ is applied to the structure connected in series with a load. Such voltage pulses are state of the art in modern semiconductor pulse power electronics. ${ }^{9}$ The passage of the tunneling-assisted impact ionization front switches the structure into the conducting state with a residual voltage of several dozens of volts. The transient $U(t)$-characteristic is nonlinear: the total duration of the switching process observed is $30 \mathrm{ps}$, whereas the effective switching time that corresponds to the major voltage drop is below 15 ps. Hence a voltage pulse of rise rate $\sim 500 \mathrm{kV} / \mathrm{ns}$ and an amplitude of several kilovolts is applied to the load. These values set new frontiers in pulse power electronics.

We finally remark, that the standard drift-diffusion model has serious limitations when applied to models of superfast ionization fronts. These limitations are due to the continuum approximation. They manifest themselves in a 
premature unphysical triggering of the front at low electrical fields due to avalanche processes initiated by tiny fractions of electrons or holes. We have eliminated these unphysical solutions by introducing a cutoff in the impact ionization term. For further progress towards a fully quantitative description, microscopic stochastic models have to be investigated.

\section{ACKNOWLEDGMENTS}

This work was supported by the Dutch physics funding agency FOM and the program "Generation of high power pulses of electrical energy, particles and electro-magnetic radiation" of the Presidium of the Russian Academy of Sciences. One of the authors (P. R.) acknowledges support of the Alexander von Humboldt Foundation.

${ }^{1}$ S. M. Sze, Physics of Semiconductor Devices (Wiley, New York, 1981).

${ }^{2}$ M. Shur, Physics of Semiconductor Devices (Prentice-Hall, Englewood Cliffs, 1990).

${ }^{3}$ M. Shaw, V. Mitin, E. Schöll, and H. Grubin, The Physics on Instabilities in Solid State Electron Devices (Plenum, New York, 1992).

${ }^{4}$ H. J. Prager, K. K. N. Chang and J. Wiesbord, Proc. IEEE 55, 586 (1968).

${ }^{5}$ B. C. Deloach and D. L. Scharfetter, IEEE Trans. Electron Devices ED20, 9 (1970).

${ }^{6}$ I. V. Grekhov and A. F. Kardo-Sysoev, Sov. Tech. Phys. Lett. 5, 395 (1979) [Pis'ma Zh. Tekh. Fiz. 5, 950 (1979)].

${ }^{7}$ D. Benzel and M. Pocha, Rev. Sci. Instrum. 56, 1456 (1985).

${ }^{8}$ Zh. I. Alferov, I. V. Grekhov, V. M. Efanov, A. F. Kardo-Sysoev, V. I. Korol'kov, and M. N. Stepanova, Sov. Tech. Phys. Lett. 13, 454 (1987) [Pis'ma Zh. Tekh. Fiz. 13, 1089 (1987)].

${ }^{9}$ I. V. Grekhov, Solid-State Electron. 32, 923 (1989)

${ }^{10}$ A. Minarsky and P. Rodin, Semiconductors 34, 665 (2000).

${ }^{11}$ R. J. Focia, E. Schamiloglu, C. B. Fledermann, F. J. Agee, and J. Gaudet, IEEE Trans. Plasma Sci. 25, 138 (1997).

${ }^{12}$ A. F. Kardo-Susoev, V. M. Efanov, and I. G. Chashnikov, in Digest of Technical Papers, 10th IEEE International Pulsed Power Conference, edited by W. L. Baker and G. Cooperstain, Albuquerque, 1995, pp. 342-347.

${ }^{13}$ V. A. Kozlov, A. F. Kardo-Susoev and V. I. Brulevsky, Semiconductors 35, 608 (2001) [Fiz. Tekh. Poluprovodn. 35, 629 (2001)].

${ }^{14}$ C. Jacobini, C. Canali, G. Ottaviani, and A. Alberigi, Solid-State Electron. 20, 77 (1977).

${ }^{15}$ Our results are not sensitive to boundary conditions. In particular, the main characteristics of the process remains the same, if homogeneous Neumann boundary conditions are used for both electron and hole concentrations at both contacts.

${ }^{16}$ E. Burstein and S. Lundqvist, eds., Tunneling Phenomena in Solids (Plenum, New York, 1969).

${ }^{17}$ V. A. Kuz'min, N. N. Krukova, A. S. Kuregyan, T. T. Mnatsakanov, and V. B. Shuman, Sov. Phys. Semicond. 9, 481 (1975) [Fiz. Tekh. Poluprovodn. 9, 735 (1975)].

${ }^{18}$ A. F. Kardo-Susoev, unpublished.

${ }^{19}$ P. Rodin, U. Ebert, W. Hundsdorfer, and I. Grekhov, J. Appl. Phys. (to be published).

${ }^{20}$ J. C. Strikwerda, Finite Difference Schemes and Partial Differential Equations (Chapman \& Hall, New York, 1989).

${ }^{21}$ J. G. Verwer, W. Hundsdorfer, J. G. Blom, Surv. Math. Ind. 10, 107 (2002); also available at Internet address http://www.cwi.nl/static/ publications/reports/abs/MAS-R9825.html

${ }^{22}$ Yu. P. Raizer, Gas Discharge Physics, 2nd ed. (Springer, Berlin, 1997).

${ }^{23}$ Under the current experimental conditions, a solitary avalanche is likely to undergo an avalanche-to-streamer transition. If this happens, it will continue to propagate essentially with saturated drift velocity, but will produce less carriers and spread less fast in the transverse direction.

${ }^{24}$ S. N. Vainshtein, Ya. V. Zhilyaev, and M. E. Levinshtein, Sov. Tech. Phys. Lett. 14, 664 (1988)

${ }^{25}$ S. N. Vainshtein, Ya. V. Zhilyaev, and M. E. Levinshtein, Sov. Phys. Semicond. 22, 717 (1988).

${ }^{26}$ S. N. Vainshtein, A. J. Kilpelä, J. T. Kostamovaara, R. A. Myllylä, S. U. Starobinets, and J. V. Zhilyaev, IEEE Trans. Electron Devices 41, 1444 (1991)

${ }^{27}$ A. Minarsky and P. Rodin, Solid-State Electron. 31, 366 (1997).

${ }^{28}$ Note, that despite of high voltage and considerable total current during the delay stage, Joule heating of the sample does not occur because the conductive component of the total current is zero.

${ }^{29}$ P. T. Landsberg, Recombination in Semiconductors (Cambridge University Press, Cambridge, 1991).

${ }^{30}$ T. I. Mnatsakanov, I. L. Rostovtzev, and N. I. Philatov, Solid-State Electron. 30, 579 (1987).

${ }^{31}$ M. I. D'yakonov and V. Yu. Kachorovskii, Sov. Phys. JETP 67, 1049 (1988) [Zh. Eksp. Teor. Fiz. 94, 321 (1988)].

${ }^{32}$ M. I. D'yakonov and V. Yu. Kachorovskii, Sov. Phys. JETP 68, 1070 (1989) [Zh. Eksp. Teor. Fiz. 95, 1850 (1989)].

${ }^{33}$ I. V. Grekhov, Vestn. Akad. Nauk SSSR 6, 18 (1981).

${ }^{34}$ O. V. Konstantinov, O. A. Mezrin, Sov. Tech. Phys. Lett. 13, 197 (1987) [Pis'ma Zh. Tekh. Fiz. 13, 476 (1987)].

${ }^{35}$ U. Ebert, W. van Saarloos, and C. Caroli, Phys. Rev. Lett. 77, 4178 (1996); Phys. Rev. E 55, 1530 (1997). 